\title{
Eine Neuedition von P.Berl. Cohen 8
}

\author{
Dieter Hagedorn und Fabian Reiter*
}

\begin{abstract}
A new edition of the sale of an ass from Kerkesoucha P.Berl. Cohen 8, offering new readings especially of the prescript, gives new weight to the text in regard to the discussion of the process of centralization of the activities of the state notaries in the Arsinoite nome at the end of the II cent. C.E.
\end{abstract}

Keywords: ass sale, Kerkesoucha, village grapheia, state

DOI 10.1515/apf-2015-0032

In einem jüngst erschienenen Aufsatz hat W. Graham Claytor die spezifische Ausgestaltung der 15 uns erhaltenen Eselkaufverträge herausgearbeitet, die im 2. Jh. n.Chr. im Grapheion des im Herakleides-Bezirk des Arsinoites gelegenen Dorfes Kerkesucha aufgesetzt worden sind; ${ }^{1}$ ihr Formular ist durch eine besondere Kürze gekennzeichnet, die sich deutlich von dem in anderen arsinoitischen Dörfern verwendeten Formular abhebt. Im Zusammenhang mit der Behandlung des auch zu der Gruppe gehörenden P.Berl. Cohen 8 aus dem Jahre 190 n.Chr. hat Claytor einige wertvolle Korrekturen zu der Edition dieses schwierigen Textes beigesteuert, aber noch nicht alle Probleme beseitigen können. Er zitiert auf S. 204 in Fußn. 25 wörtlich eine Feststellung, die Fritz Mitthof im Jahre 2008 getroffen hat, nämlich: „Das einzigartige Präskript der Urkunde harrt noch einer überzeugenden Deutung“. ${ }^{2}$ Dies hat uns daran erinnert, daß wir uns bereits im Oktober 2007 in einem über E-Mail geführten Gedankenaustausch mit ebendiesem Präskript befaßt haben und glaubten, dabei - zumal aufgrund

\footnotetext{
*Corresponding authors: Dieter Hagedorn, Kirschblütenweg 6, D-50996 Köln, <dieter. hagedorn@urz.uni-heidelberg.de>; Fabian Reiter, Universität Trier, Fachbereich III, Papyrologie, 54286 Trier, <reiterf@uni-trier.de>

${ }^{1}$ W. Graham Claytor, Donkey Sales from the grapheion of Kerkesoucha, ZPE 194 (2015) 201-208.

${ }^{2}$ Fritz Mitthof, Urkundenreferat 2007 (1. Teil), APF 54 (2008) 266-298, hier S. 269.
} 
einer Autopsie durch Fabian Reiter - wesentliche Fortschritte in der Entzifferung gemacht $\mathrm{zu}$ haben. Zu der geplanten Veröffentlichung unserer Erkenntnisse ist es jedoch nicht gekommen, unter anderem, weil wir die verbliebenen, inzwischen von Claytor gelösten Probleme als ein Hindernis empfunden haben. Jetzt scheint es uns an der Zeit, den Faden wieder aufzunehmen. Wir haben den Text anhand der digitalen Abbildung, die mittlerweile im Internet zur Verfügung steht, ${ }^{3}$ erneut überprüft und uns aufgrund der dabei gewonnenen Erkenntnisse - mögen auch einzelne problematische Stellen weiterhin ungelöst bleiben - entschlossen, den unten folgenden vollständigen Neudruck vorzulegen.

In seiner neuen Gestalt wirft der Text ein Schlaglicht auf den Ablauf des insbesondere von Hans Julius Wolff beschriebenen Prozesses, in dessen Verlauf die Dorfgrapheia im Arsinoites - offenbar zu unterschiedlichen Zeitpunkten oder -phasen - zugunsten eines einzigen zentralen Staatsnotariats in der Metropole aufgegeben worden sind, ${ }^{4}$ und gibt dabei einen interessanten Etappenschritt zu erkennen; denn drei Zeugnisse aus dem Zeitraum von 188-190 n.Chr. scheinen zu beweisen, daß die Verwaltung aller Dorfgrapheia und des metropolitischen Staatsnotariats in nur einer Hand lag, der eines Beamten, dessen Funktion als die eines

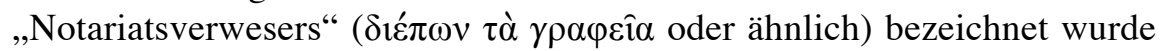
(s. unten zu Z. 4-7).

Akzeptiert man diesen Schluß, so tauchen bei der Weiterführung des Gedankens zahlreiche Fragen und Probleme auf, darunter folgende: Die Formulierungen in den drei Texten zeigen einerseits klar, daß jener Notariatsverweser gauweit alle Schreibämter führte, weswegen man seinen Amtssitz in der Metropole vermuten würde, gleichzeitig steht aber das Formular des Eselkaufs in P.Berl. Cohen 8 eindeutig in der Tradition des Grapheions von Kerkesucha, wird also gewiß dort abgefaßt worden

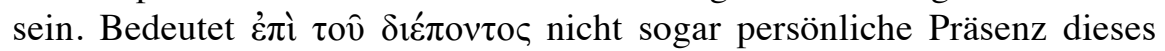
Notariatsverwalters? Dann müßte man annehmen, daß er im Gau umhergereist sei und etwa turnusmäßige Sprechstunden an den wichtigen Notariatsorten hatte, die zu dieser Zeit vielleicht nur noch wenige waren.

\footnotetext{
${ }^{3}$ S. <http://smb.museum/berlpap/index.php/15896/>; vgl. auch die Schwarz-Weiß-Abbildung in der Erstedition, P.Berl. Cohen, Tafel 8.

${ }^{4}$ Hans Julius Wolff, Das Recht der griechischen Papyri Ägyptens in der Zeit der Ptolemaeer und des Prinzipats, II, München 1978, S. 18-23, bes. 21 .
} 
Ist es nicht seltsam, daß sein Name nicht genannt ist und sich am Ende kein Notarsvermerk findet? ${ }^{5}$

Die Klärung dieser und anderer verwandter Fragen erforderte eine gründliche Durchsicht aller Zeugnisse für die arsinoitischen Grapheia und auch der in der Metropole geschlossenen Verträge, zu der wir uns gegenwärtig nicht in der Lage sehen.

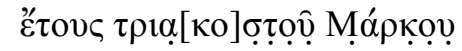

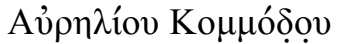

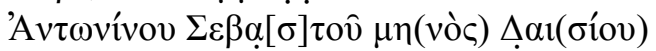

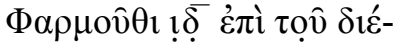

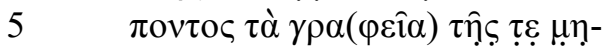

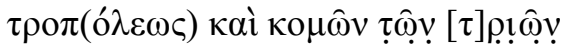

$\mu \varepsilon \rho i ́ \delta \omega \nu \ldots \ldots . \dot{\varepsilon} v$

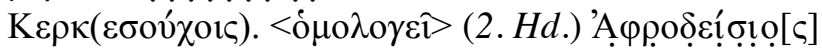

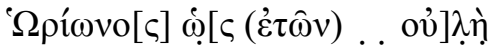

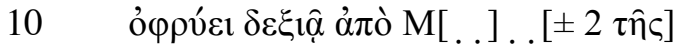

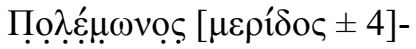

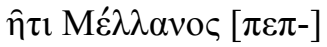

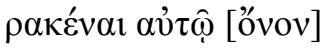

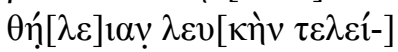

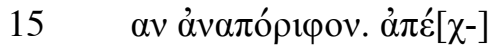

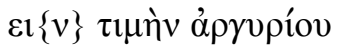

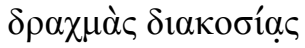

$\tau \varepsilon \sigma \sigma \varepsilon \rho \alpha ́ \kappa о v \tau \alpha$.

$\beta \varepsilon \beta \varepsilon \circ \hat{\imath}$.

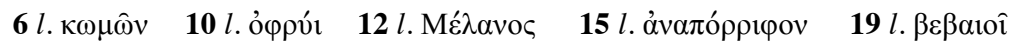

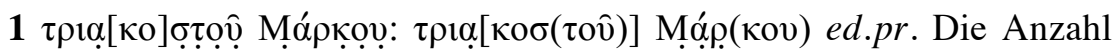
der (wenigstens in Spuren) noch vorhandenen Buchstaben hat der Herausgeber deutlich unterschätzt. Bei der Identifizierung der Tintenreste bleibt allerdings vieles unsicher.

${ }^{5}$ Eine Randbemerkung: In der Parallele für einen staatsnotariellen Eselkauf aus Kerkesucha BGU XV 2480 (= Nr. 4 bei Claytor, a.a.O.) scheint in Z. 29 der erwartete Registrierungsvermerk (vgl. den Kommentar der Edition) halbwegs lesbar zu sein; zumindest

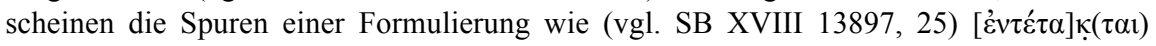

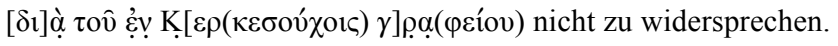




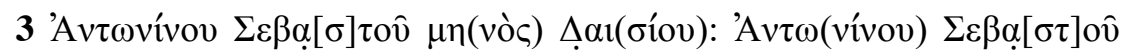

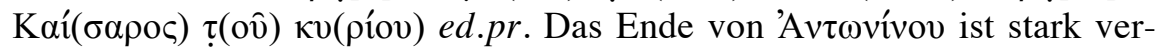
schliffen. Die vom Herausgeber angenommene Verbindung $\Sigma \varepsilon \beta \alpha \sigma \tau \grave{c} \varsigma$

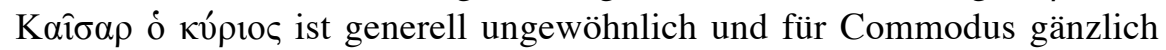
unbezeugt. ${ }^{6}$ Zur Verwendung der makedonischen und ägyptischen Monatsnamen nebeneinander vgl. Ursula Hagedorn, Gebrauch und Verbreitung makedonischer Monatsnamen im römischen Ägypten, ZPE 23 (1976) 143-167, wo nachgewiesen wird, daß dies in römischer Zeit eine Eigentümlichkeit in Staatsnotariatsurkunden ist, d.h. der Vertragsform, die auch hier vorliegt (s. auch unten). Das Eta von $\mu$ (vóc) ist vom Typ 4 (ähnlich, allerdings von anderer Hand, in Z. $16 \tau \mu \mu \eta$ v) und hat einen sehr weit nach unten reichenden Abstrich; auch das Iota von $\Delta \alpha 1(\sigma i ́ o v)$ reicht tief nach unten, was ein Anzeichen für Abkürzung sein könnte. Danach sind

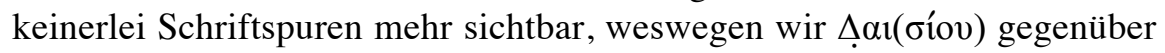
der Ergänzung $\Delta \alpha_{1}[\sigma i ́ o v]$, die wir auch in Erwägung gezogen haben, vorziehen.

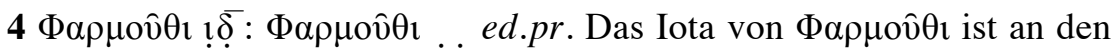
Querstrich des Theta angebunden, das folgende Iota verläuft parallel dazu. Das Datum entspricht dem 9. April 190. Anstelle von $\underline{\underline{\delta}}$ erscheint uns ein einzelnes $\eta^{-}(=3$. April $)$ weniger wahrscheinlich.

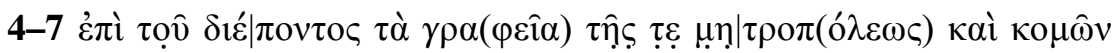

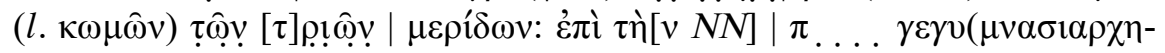

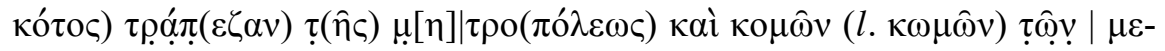

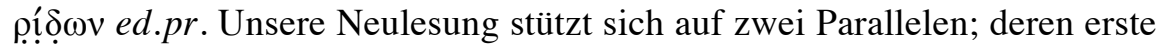
liefert das Testament, welches in Abschrift in P.Hamb. IV 278 (Arsinoites; 30. September 190) vorliegt und dessen Präskript der Edition zufolge

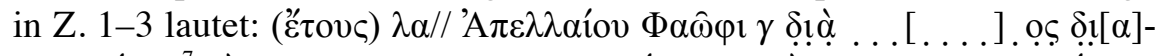

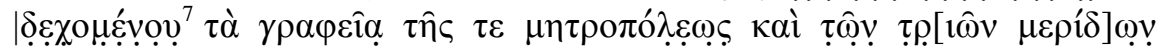

\footnotetext{
${ }^{6}$ In P.Bour. 27, 2 (187/8 n.Chr.), wo diese Abfolge durch Ergänzung hergestellt worden ist, muß ebenfalls korrigiert werden, und zwar durch einfache Tilgung des Bestandteils

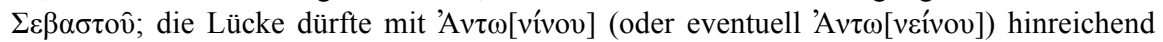
gefüllt sein.

${ }^{7}$ Im Gegensatz zu der im Kommentar zu P.Hamb. IV 278, 1-3 getroffenen Feststel-

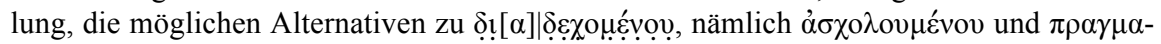
$\tau \varepsilon v o \mu \varepsilon ́ v o v$, seien weniger gut mit den Spuren vereinbar, halten wir jetzt an der digitalen Abbildung $\pi \rho \alpha \gamma \mu \alpha \tau \varepsilon v o \mu \varepsilon ́ v o v$ doch für nicht völlig unmöglich, und zwar durch den Vergleich mit Z. 28 - $\tau \varepsilon v o \mu \varepsilon ́ v o r \varsigma$ (s. <http://wwwapp.cc.columbia.edu/ldpd/app/apis/item?

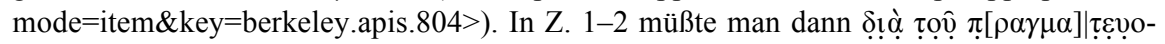
ب̣̂́ṿọ schreiben.
} 


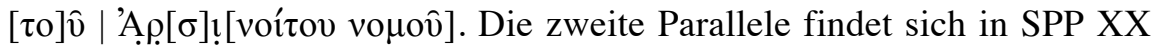
14, einem Antrag auf Eröffnung eines Testaments (Arsinoites; 17. Juni 188), der nach dem Verlust des Anfangs in Z. 1-3 mitten in der Erwähnung des Notars beginnt, durch den das Testament erstellt worden ist:

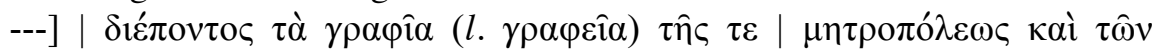

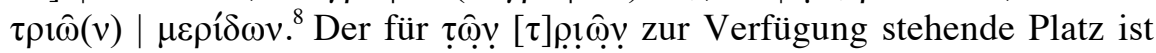
zugegebenermaßen sehr gering; wir erwägen alternativ stattdessen $\tau \hat{\omega} \varphi \gamma^{\prime}$.

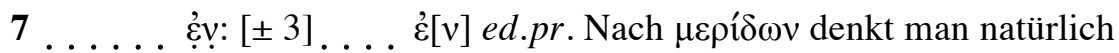

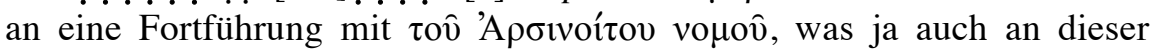
Stelle in P.Hamb. IV 278 hergestellt worden ist, allerdings nahezu vollkommen ergänzt. In P.Berl. Cohen 8 kann diese Wendung jedoch keinesfalls gestanden haben, und für gleichfalls unmöglich halten wir, daß in Z. 6-7 દ’v $\dot{\alpha} \gamma_{0} \rho \hat{\alpha}|\mathrm{K}| \mathrm{K} \rho \kappa(\varepsilon \sigma o v ́ \chi \omega v)$ gestanden haben könnte, vgl. den Eselkaufvertrag BGU II $413=$ M.Chr. 263, 4 (3. Febr. 219) ${ }^{9}$. Der erste der ungelesenen Buchstaben erinnert uns an ein $\mu$, und die letzten drei wären eventuell als $\lfloor! ̣$ zu deuten. Wir sehen uns außerstande, einen Lesungsvorschlag zu unterbreiten.

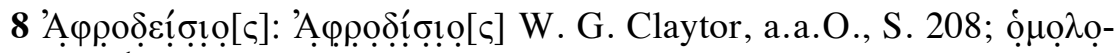

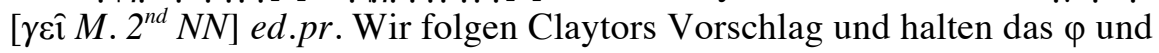
das erste o sogar für so sicher, daß sie keines Punktes bedürfen. Außerdem glauben wir, nach dem $\delta$ deutlich $\varepsilon$ zu sehen, an das in Ligatur $!$

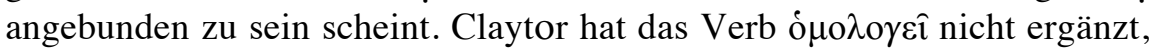
sondern für sein Fehlen darauf verwiesen, daß „Bank contracts are frequently drawn up without an opening main verb“. Da allerdings nach

\footnotetext{
${ }^{8}$ Mehrere Korrekturen zu diesem Text hat Fritz Mitthof in Tyche 17 (2002) 257f. mit-

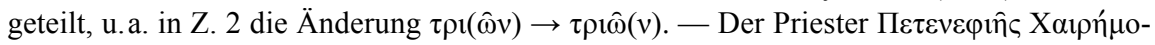
voc, der in SPP XX 14 den Antrag stellt (s. Z. 12), könnte ein Nachkomme des Priesters

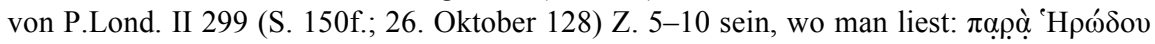

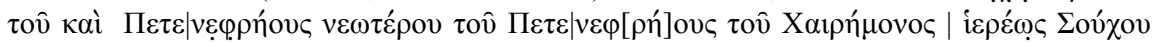

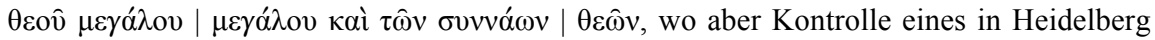

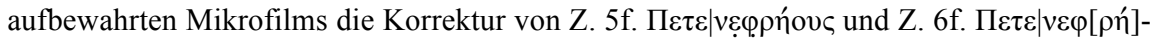

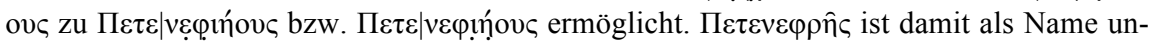

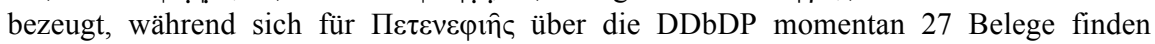
lassen.

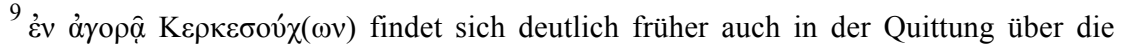
Steuer für den Verkauf einer Kuh P.Fay. 62,3. Wir nehmen die Gelegenheit wahr, darauf aufmerksam zu machen, daß nach Ausweis der Abbildung im Internet (s. dazu das HGV)

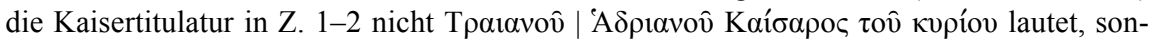

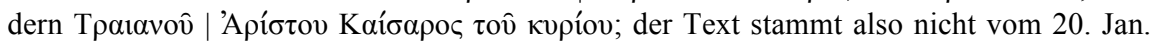
134, sondern vom 20. Jan. 115 n.Chr.
} 
unserer Neulesung jetzt das Wort $\tau \rho \alpha ́ \pi \varepsilon \zeta \alpha$ in Z. 5 verschwunden ist, liegt der Gedanke an eine Bankurkunde nicht mehr nahe, sondern es ist klar, daß wir es mit einer Staatsnotariatsurkunde zu tun haben. Wir schlagen eine andere Erklärung vor: Claytor hat in seinem Aufsatz dargelegt, daß in den Eselkaufverträgen aus Kerkesucha die in der Regel von einem ersten Schreiber vorgefertigten Vertragsanfänge gewöhnlich bis einschließlich zu

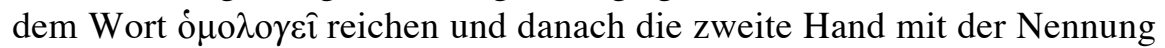
des Verkäufers einsetzt. In diesem Fall hat der erste Schreiber offenbar ó ${ }_{0} \lambda$ o $\varepsilon \hat{\imath}$ weggelassen (vielleicht weil er die Möglichkeit eines eventuell

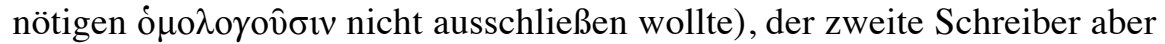
hat, ohne die Abweichung vom Üblichen zu bemerken, routinemäßig seine Arbeit mit dem Namen des Verkäufers begonnen.

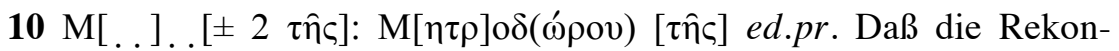
struktion der ed.pr. zu unsicher ist, weil o $\delta$ nicht zweifelsfrei zu lesen ist und folglich andere Ergänzungen in Frage kommen, hat mit Recht Claytor a.a.O., S. 208 hervorgehoben. In der Polemonos Meris lagen z.B. auch

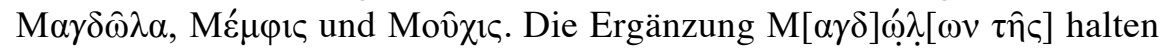

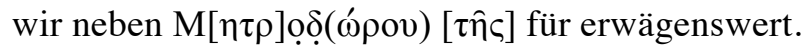

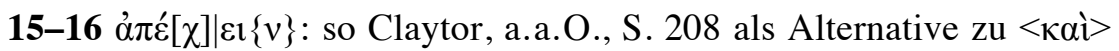

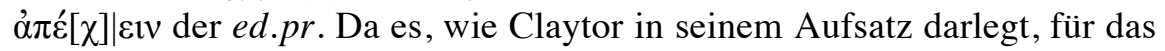
Formular der aus Kerkesucha stammenden Verträge typisch ist, daß die Formel asyndetisch mit dem finiten Verb eingeführt wird, sollte man dieser Lösung den Vorzug geben.

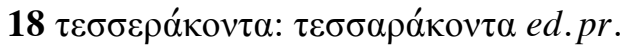

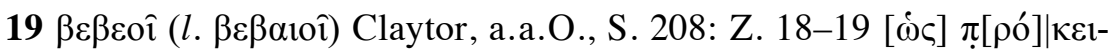
$\tau \alpha \mathrm{l}$ ed.pr. Daß die Garantieklausel, radikal verkürzt, aus einem isolierten $\beta \varepsilon \beta \alpha$ เô̂ besteht, ist ebenfalls ein Spezifikum der einschlägigen Verträge aus Kerkesucha. 